\title{
Shared care for chronic eye diseases: perspectives of ophthalmologists, optometrists and patients
}

\section{Patricia M O'Connor Research Fellow \\ C Alex Harper MB BS, FRANZCO Ophthalmologist \\ Cathy L Brunton \\ BNurs, \\ Improvement and Innovation Manager \\ Sandra J Clews \\ Ophthalmic Nurse and Project Coordinator \\ Sharon A Haymes BScOptom, PhD, MPH \\ Research Fellow and Optometrist, 1,2 \\ Jill E Keeffe $\mathrm{OAM}, \mathrm{PhD}$ Head, Populatio Health Unit ${ }^{1,2}$ \\ 1 Centre for Eye Research Australia, University of Melbourne Melbourne, VIC \\ 2 Royal Victorian Eye and Ear Hospital Melbourne, VIC \\ jillek@unimelb.edu.au}

MJA 2012; 196: 646-650 doi: 10.5694/mjall.10856 mproving access to eye health care services is a key action area in Australia's National Eye Health Framework. ${ }^{1}$ Three chronic eye diseases that are among the top five causes of vision impairment in Australia are age-related macular degeneration (AMD), diabetic retinopathy (DR) and glaucoma. ${ }^{2}$ These diseases are managed mainly by ophthalmologists, while optometrists in primary care have a role in case detection and monitoring, and general practitioners refer patients who may require eye care.

A major inefficiency of the current system is that not all people referred for ophthalmological care require services or ongoing treatment in a secondary or tertiary setting. Most patients with these chronic eye diseases do not require treatment if the condition is stable. As the predominant need is for monitoring, shared care arrangements with optometrists could yield considerable patient and service benefits. This has been shown to be the case in the United Kingdom $^{3-5}$ and United States. ${ }^{6}$

In Australia, the optometry workforce (3329 full-time equivalents [FTE]) exceeds that of ophthalmologists (735 FTEs). ${ }^{7}$ This, coupled with their greater geographical coverage in rural and urban locations, offers the potential to provide patients in the early stages of eye disease with local care, addressing issues of transport, cost and access that are known barriers to eye care service uptake. ${ }^{3}$

Some developments in shared care have already occurred in Australia. Current National Health and Medical Research Council guidelines encourage detection and monitoring of DR by optometrists, and the establishment of networks between optometrists and ophthalmologists to monitor glaucoma. ${ }^{8,9}$ Guidelines introduced in 2008 authorise optometrists with postgraduate qualifications in ocular therapeutics to prescribe pharmacological agents for glaucoma

\begin{abstract}
Ahstrac
Objective: To report the perspectives of optometrists, ophthalmologists and patients on a model of shared care for patients with chronic eye diseases.

Design, setting and participants: Qualitative study of a model of shared care between optometrists and ophthalmologists for patients with stable agerelated macular degeneration, diabetic retinopathy and glaucoma, trialled by the Royal Victorian Eye and Ear Hospital in Melbourne during 2007-2009. Semistructured interviews were conducted with optometrists, ophthalmologists and patients at completion of the project to obtain their perspectives on this model.

Results: Seventeen optometrists submitted expressions of interest to participate, and 12 completed web-based training modules and clinical observerships and adhered to specified examination and reporting protocols. All five participating ophthalmologists and 11 of the optometrists were interviewed. Ninety-eight patients participated and 37 were interviewed. Optometrists not only met ophthalmologists' expectations but exceeded them, appropriately detecting and referring patients with additional, previously undetected conditions. Patients reported savings in travel time and were satisfied with the quality of care they received. Optometrists, ophthalmologists and patients indicated a general acceptance of shared care arrangements, although there were some issues relating to interprofessional trust.

Conclusions: Shared care between local optometrists and hospital-based ophthalmologists can help to reduce patient waiting time for review and offers an opportunity for these two groups of eye care professionals to collaborate in providing localised care for the benefit of patients. However, trust and relationship building need to be further developed.
\end{abstract}

patients and to be involved with ophthalmologists in developing written patient management plans. ${ }^{10}$ To date, $14.8 \%$ of optometrists in Australia have become therapeutically endorsed. ${ }^{11}$ Despite these developments, the full potential for shared care has not been fully realised.

Here, we report the perspectives and experiences of optometrists, ophthalmologists and patients involved in a model of shared care trialled by the Royal Victorian Eye and Ear Hospital (RVEEH) in Melbourne.

\section{Methods}

This 2-year National Eye Health Demonstration Project, conducted during 2007-2009, involved shared care between the RVEEH and community eye care practitioners. Its aims were to reduce the number of patients with AMD, DR and glaucoma waiting for review appointments and to engage primary care providers in monitoring these diseases, thereby increasing patient convenience and ensuring appropriate hospital referrals.

Expressions of interest were sought from registered optometrists in six urban areas of metropolitan Melbourne and two rural areas of Victoria (Hume and Gippsland), selected on the basis of patient distributions. ${ }^{12}$ Advertisements were placed in newsletters of the Optometrists Association Australia (OAA) and the Australian College of Optometry (ACO), and on the RVEEH website. To be eligible to participate, optometrists were required to possess and be competent in using specified equipment (Box 1), complete project-specific web-based training modules and RVEEH clinical observerships, and adhere to standardised patient examination and reporting protocols.

For each of the three eye diseases, a web-based module and patient care protocol were developed under the direction of the lead ophthalmologists, using available guidelines. ${ }^{8,13,14}$ Each module comprised a knowledge 
section (disease characteristics, patient monitoring and referral criteria) and an assessment section with images and questions (10 multiplechoice questions on DR, 26 multiplechoice and eight open-ended questions on AMD, and an online interactive glaucomatous optic neuropathy grading test ${ }^{15}$ ).

The AMD and DR clinical observerships each involved two visits to RVEEH clinics of 2.5 hours' duration. The glaucoma observership required attendance at a 2-hour workshop where each optometrist examined the same 11 patients and discussed findings as a group with the lead ophthalmologist.

The project was rolled out in stages to accommodate the logistics of completing the web modules and observerships for each disease.

A project manager and an ophthalmic nurse coordinator (S J C) undertook day-to-day governance of the project at the RVEEH. A steering committee was established, comprising key stakeholders from the RVEEH, OAA, ACO, Melbourne General Practice Network, Australian Government Department of Health and Ageing, Victorian Department of Health,
Orthoptic Association of Australia, Australian Ophthalmic Nurses Association and Centre for Eye Research Australia. Five ophthalmologists provided clinical oversight. Ophthalmologists and optometrists received no remuneration for participating.

Lists of patients who had been awaiting review appointments for 12 months or more and who lived within the catchments of participating optometrists were obtained for each eye disease. The coordinator reviewed files to identify patients who met the eligibility criteria (Box 2). Once approved by an RVEEH ophthalmologist, the coordinator approached eligible patients and offered them the option of ongoing monitoring by a participating local optometrist. Patients' reasons for declining were recorded.

On completion of the project, semistructured interviews were conducted to obtain patients' and clinicians' perspectives of the model. Interview questions were designed to reflect the project objectives and were developed through an iterative process involving stakeholder discussion and inputs and endorsement at steering committee level.
1 Equipment required for optometrists' participation in the project

- Slit lamp and either a 78 or 90 dioptre lens

- Amsler grid

- Contact applanation tonometer (in some circumstances, a non-contact tonometer may be used)

- Optional: threshold automated perimetry, preferably using a perimeter with the capability of objectively comparing the results of two different field plots

The sample of patients selected for telephone interview was representative of each eye disease and geographical location, and included both patients who participated and patients who declined. To ensure consistency in administration and data collection, the same researcher administered the standard interview schedule and transcribed patients' responses to questions on decision making, self-reported vision difficulties, travel issues, whether they were currently attending an optometrist and whether they had any concerns about attending the RVEEH-nominated optometrist. Patients were also asked to rate the quality of service received on a scale from 1 (poor) to 6 (excellent) and to indicate their willingness to continue with shared care.

One of us ( $\left.\mathrm{P} \mathrm{O}^{\prime} \mathrm{C}\right)$, a program evaluator (not an ophthalmologist or

\section{Patient eligibility criteria, by eye disease}

Inclusion criteria

Age-related macular degeneration (AMD)
prog-exudative AMD - increased number of drusen,
Pure geographic atrophy - in the absence of exudative
AMD or loss of RPE and increased visualisation of the
underlying choroid, and with no change to symptoms
Diabetic retinopathy (DR)
No DR or minimal non-proliferative DR
Glaucoma
a) Ocular hypertension / glaucoma suspect
IOP above 22 mmHg with full visual field
No abnormalities in colour, size, shape, neuroretinal rim,
cup-to-disc ratio, retinal arteries and nerve fibre layer for
age, no disc haemorrhages, no peripapillary atrophy
Peripheral iridotomies may be present
b) Physiological cupping
- IOP $\leqslant 21$ mmHg
No abnormalities in colour, size, shape, neuroretinal rim,
age, no disc haemorrhages, no peripapillary atrophy
Open angles

Exclusion criteria

- Non-exudative AMD - high-risk patients with increased number of drusen, progressive areas of atrophy, VA 20/25 and symptoms

- Exudative AMD with RPE or serous detachment of sensory retina

- Subretinal or pigment epithelium haemorrhage

- Mild, moderate or severe non-proliferative DR, proliferative DR and high-risk proliferative DR

- Glaucoma diagnosis

- Receiving treatment for glaucoma

- Visual field defects

- $\mathrm{IOP} \geqslant 22 \mathrm{mmHg}$

- Asymmetry in shape and size of the optic nerves between the two eyes

- Glaucoma diagnosis

- Receiving treatment for glaucoma

- Visual field defects

- Occludable angles 
3 Comparison of demographic characteristics of patients invited to participate*

\begin{tabular}{lcc} 
Characteristic & $\begin{array}{c}\text { Accepted } \\
(n=103)\end{array}$ & $\begin{array}{c}\text { Declined } \\
(n=76)^{\dagger}\end{array}$ \\
\hline Age, years & $70 \pm 13$ & $70 \pm 11$ \\
Mean \pm SD & $35-93$ & $40-94$ \\
Range & & \\
Sex & $49(48 \%)$ & $39(51 \%)$ \\
Male & $54(52 \%)$ & $37(49 \%)$ \\
Female & $53(51 \%)$ & $52(68 \%)$ \\
Eye condition & $13(13 \%)$ & $6(8 \%)$ \\
Diabetic retinopathy & $22(21 \%)$ & $13(17 \%)$ \\
Age-related macular degeneration & $15(15 \%)$ & $5(7 \%)$ \\
Glaucoma & & \\
Multiple conditions ${ }^{\ddagger}$ & $101(98 \%)$ & $75(99 \%)$ \\
Location & $2(2 \%)$ & $1(1 \%)$ \\
\hline Urban & & \\
Rural & &
\end{tabular}

* Differences between patients who accepted and those who declined participation were not statistically significant $(P>0.05)$. †Comprising 69 patients who declined and seven who deferred decision making. $\ddagger$ Any combination of the three eye conditions. selection. Nine of the 12 participating optometrists were from ACO public clinics. Distance of optometry practices from the RVEEH ranged from 2 to $50 \mathrm{~km}$ for urban sites, and 163 to $281 \mathrm{~km}$ for rural sites.

\section{Patient management}

Of 1091 patients initially identified, 251 met the eligibility criteria. Further investigation found 58 (23\%) of these had already been reviewed or held review appointments, three had died, three were no longer RVEEH patients, and eight could not be contacted. The remaining 179 patients were approached (mean age \pm SD, $69.9 \pm 11.9$ years; 51\% female). Of these, 103 (58\%) agreed to participate, 69 declined and seven deferred making a decision (Box 3).

The staged roll-out of the project meant that the majority of the 103 participants had DR $(53 ; 51 \%)$, as this was the first disease group to start. Participants were mainly from urban areas; only two participants attended rural optometrists. Thirty participants (29\%) attended a single site (ACO Carlton) and $62(60 \%)$ attended other ACO optometrists.

Of the 103 participating patients, 98 completed the process, while two voluntarily resumed care at the RVEEH, three decided to seek private care and one died. Eighty-four patients remained eligible for shared care at the end of the project and six were discharged by the RVEEH. The remainder resumed ongoing care at RVEEH as a result of optometrists having detected onset or progression of disease or a need for cataract surgery and referring them back to the RVEEH - these decisions were endorsed by the ophthalmologists.

\section{Clinician perspectives}

Eleven optometrists and all five ophthalmologists were interviewed. While optometrists generally understood the need to have some formal entry system to participate, they questioned the level of assessment required: "the sense of being assessed and accredited in areas that I've worked in for years didn't seem right" and the "methods [used] were presumptive of a negative ability of optometrists". Ophthalmologists considered the selection criteria appropriate, and most described themselves as having been "cautious" in confirming practitioners.

For optometrists, the convenience of undertaking the web training modules in their own time and off site was a positive aspect. However, the webbased assessment was not considered representative of clinical practice or diagnostic abilities, as only still images and limited case findings were provided. Ophthalmologists also recognised the limitations of the web modules without reviewing practice in a clinical setting.

Ophthalmologists and optometrists both valued the relationship-building opportunities provided by the face-toface observerships. Indeed, relationship building was a major motivating factor for optometrists' involvement. Observing RVEEH management protocols and gaining exposure to new treatments and to more severe cases were further positive outcomes for optometrists.

However, ophthalmologists and optometrists were both concerned about how time-consuming the observerships were, with lost clinic time an issue for both groups. Nonetheless, some ophthalmologists thought more time was needed to thoroughly observe the optometrists' diagnostic skills.

Optometrists considered the project "conservative in [its] choice of patients", that "the level of expertise needed to manage patients was very low" and that they had hoped to be assigned "more complex patients". Additionally, rural optometrists were disappointed that their expectations of getting "a steady trickle [of patients] didn't happen".

The administrative role undertaken by the coordinator on behalf of ophthalmologists and the standardised reports required from optometrists resulted in practitioners reporting minimal administrative burden.

\section{Patient perspectives}

A total of 46 patients were interviewed: 37 participants and nine nonparticipants. Twenty-five of the participants listed direct contact from the RVEEH as their main reason for choosing shared care. Of all 46 patients interviewed, only five (two of whom were participants) had any 
concerns with attending the suggested optometrist. Four non-participants and 11 participants had an optometrist they usually attended.

Most interviewed participants (30/ 37) found travel to the local optometrist easier than travel to the RVEEH; three considered it more difficult, and four reported no major differences.

The interviewed non-participants listed ease of transport (5/9), reputation of the RVEEH (2/9), familiarity with the RVEEH (1/9), and not fully understanding the introductory letter (1/9) as their main reasons for not participating. Eight added that if the nominated optometrist had been located closer to them, they might have accepted.

Similarly, access to public transport (25/69) and reputation of and familiarity with RVEEH services (17/69) were the main reasons given at the time of recruitment for declining participation.

Shared care yielded substantial savings in travel time for participants, with 30 of the 37 interviewed being located within 30 minutes and the remainder less than 1 hour from the nominated optometrist. In contrast, only 12 interviewed participants were within 30 minutes' travel of the RVEEH, 14 were within 1 hour and the remainder were within 3 hours. A significant association was found between participation and travel time $\left(\chi^{2}=17.56 ; P=0.007\right)$, with higher acceptance rates among those located furthest from the RVEEH.

Patient satisfaction with the quality of community-based care was high, with 30 of 37 participants rating the quality of service 6 or 5 , five rating it 4 and one rating it 3 . Most (34/37) indicated that they would be happy to continue having routine appointments with their project-nominated optometrist.

\section{Discussion}

The shared care model used in this project represents a solution to managing hospital demand for patients with stable AMD, DR and glaucoma. Participating ophthalmologists, optometrists and patients indicated a general openness to such arrangements, subject to a number of issues being addressed.

Perhaps the most fundamental issue was interprofessional trust. The requirement for optometrists' knowledge and diagnostic skills to be assessed, and the choice of assessment adopted by the ophthalmologists, strongly points to this. This situation is not unique to eye care. Interprofessional tensions have been observed between GPs and nurses ${ }^{17}$ and between doctors and pharmacists, $^{18}$ for example.

The ophthalmologists were in the difficult position of selecting patients for participation based on past medical records, without examining the patients themselves. They were also required to entrust patient care to optometrists with whom they mostly had no prior working relationship. To ensure satisfactory care, the ophthalmologists imposed formal, time-consuming assessments of optometrists' knowledge and skills as part of the practitioner selection process.

However, the optometrists met, and indeed exceeded, ophthalmologists' expectations, detecting and appropriately referring patients found to have new abnormalities. The appropriateness of these referrals, verified by the ophthalmologists' endorsement, signifies that, unlike optometrists in previous studies, these practitioners' referrals were not overly conservative. ${ }^{19}$

Trust and relationship building would do much to ameliorate the concerns of the ophthalmologists and the frustration expressed by optometrists that their skills were not adequately acknowledged or used. Direct referral of patients from hospital clinics to optometrists would address ophthalmologists' concerns about patient selection, eliminate the need for an ongoing coordinator and potentially make shared care an integral part of the service pathways considered by hospital ophthalmologists. However, without time invested in relationship building, ophthalmologists' reservations could be an ongoing impediment to implementing shared care. Inter- and intra-professional reflection is key to achieving attitudinal change. ${ }^{20}$

From the patients' perspective, shared care offers the benefits of reducing demand for review appointments at the RVEEH and the opportunity to have routine monitoring undertaken closer to home. Consistent with previous studies, ${ }^{3,21}$ distance and transport were key considerations in patients' decisions to accept or decline community-based care.

The patients' willingness to consider shared care is encouraging and suggests they did not perceive optometrists as "high street opticians they visit when they need glasses"; nor did they prefer being seen by an ophthalmologist, as found elsewhere. ${ }^{21}$ Including additional sites and mainstreaming shared care into the hospital's practice would increase local optometrist accessibility and make shared care truly "local" for more patients.

A limitation of this study is the generalisability of its findings. As a time-bound demonstration project, it involved a small group of volunteers from Victoria's 730 employed optometrists. ${ }^{7}$ Patient numbers were relatively low because participation was voluntary rather than determined by routine clinic-based decisions. Restricted geographical coverage and stringent clinical criteria further limited the number of optometrists and patients who could be involved.

For optimum efficiency, trust and relationship building must also include GPs, to achieve appropriate direct referrals to local optometrists rather than to tertiary outpatient clinics. An interprofessional collaboration project between $\mathrm{ACO}$ and the RVEEH is an important development - a pilot ACO clinic aimed to assess 1000 RVEEH GP-referred patients between October 2011 and March 2012.

Shared care between local optometrists and hospital-based ophthalmologists provides a solution to reducing hospital demand, while offering an opportunity for these two groups of eye care professionals to collaborate in providing localised care for the benefit of patients. However, for broader shared care to occur, it will be necessary to collaboratively develop standards, and to nurture partnerships and interprofessional trust, so that patient choice is enhanced and safe care assured. 
Acknowledgements: This 2-year National Eye Health Demonstration Project was funded by the Australian Government Department of Health and Ageing, with additional funds from the Victorian Department of Health. This research was supported by the Royal Victorian Eye and Ear Hospital. The Centre for Eye Research Australia receives operational infrastructure support from the Victorian Government. We acknowledge the support and cooperation of all the ophthalmologists, optometrists, support staff and patients involved, and the contribution of the project manager. Bich Thai.

Competing interests: No relevant disclosures.

Received 7 Jul 2011, accepted 20 Oct 2011.

1 Department of Health and Ageing. National framework for action to promote eye health and prevent avoidable blindness and vision loss. Canberra: Commonwealth of Australia, 2005. http://www.health.gov.au/internet/main/ publishing.nsf/Content/ageing-eyehealthframework-toc.htm (accessed Apr 2012)

2 Access Economics. Clear insight. The economic impact and cost of vision loss in Australia. Melbourne: Centre for Eye Research Australia, 2004. http://www.cera.org.au/uploads/CERA clearinsight.pdf (accessed Apr 2012).

3 Bhargava JS, Bhan-Bhargava Foss AJ, King AJ. Views of glaucoma patients on provision of follow-up care; an assessment of patient preferences by conjoint analysis. $\mathrm{Br} J$ Ophthalmol 2008; 92: 1601-1605.

4 Banes MJ, Culham LE, Bunce C, et al. Agreement between optometrists and ophthalmologists on clinical management decisions for patients with glaucoma. Br J Ophthalmol 2006; 90: 579-585.

5 Gray SF, Spry PGD, Brookes ST, et al. The Bristol Shared Care Glaucoma Study: outcome at follow up at 2 years. $\mathrm{Br} J$ Ophthalmol 2000; 84 : 456-463.

6 Myers RI. Interprofessional strategies for optometry and ophthalmology in the future. Optom Vis Sci 1995; 72: 42-44.

7 Long R. Eye health labour force in Australia. Canberra: Australian Institute of Health and Welfare, 2009. (AlHW Cat. No. PHE 116.)

8 National Health and Medical Research Council. Guidelines for the management of diabetic retinopathy. Canberra: Commonwealth of Australia, 2008. http://www.nhmrc.gov.au/ publications/synopses/dil5syn.htm (accessed Jun 2011)

9 National Health and Medical Research Council. Guidelines for the screening, prognosis, diagnosis, management and prevention of glaucoma. Canberra: Commonwealth of Australia, 2010. http://www.nhmrc.gov.au/publications/ synopses/cpll3syn.htm (accessed Jun 2011)

10 Lu CY, Lu VH, Goldberg I, Day RO. Towards integrated care: Australia's new model of care for patients with glaucoma [editorial]. Med J Aust 2010; 193: 200-201.

11 Keily PM, Horton P, Chakman J. The Australian optometric workforce 2009. Clin Exp Optom 2010; 93: 330-340.

12 Lane C, Michel K. Community mapping project report: mapping communities using the Eye and Ear Hospital and identifying the needs of Victoria's changing population. Melbourne: Royal Victorian Eye and Ear Hospital, 2008. http://www.cera.org.au/uploads/CERA_RVEEH Community_Mapping_Report.pdf (accessed Jun 2011).

13 Association of Optometrists. Primary care resource pack: glaucoma - draft protocol. 2002 http://www.assoc-optometrists.org/uploaded files/pdf/draft protocol.pdf (accessed Jun 2011; no longer available)

14 American Optometric Association. Optometric clinical practice guideline: care of the patient with age-related macular degeneration. St Louis, Mo: AOA, 2004. http://www.aoa.org/ documents/CPG-6.pdf (accessed Jun 2011).

15 Kong YX, Coote MA, O'Neill EC, et al. Glaucomatous optic neuropathy evaluation project: a standardized internet system for assessing skills in optic disc examination. Clin Experiment Ophthalmol 2011; 39: 308-317.

16 Corbin JM, Strauss AL. Basics of qualitative research: techniques and procedures for developing grounded theory. 3rd ed. Thousand Oaks, Calif: Sage, 2008.

17 Speed S, Luker KA. Getting a visit: how district nurses and general practitioners 'organise' each other in primary care. Sociol Health Illn 2006; 28 883-902.

18 Liaw ST, Peterson G. Doctor and pharmacist back to the apothecary! Aust Health Rev 2009 33: 268-278.

19 Wright SE, McKay R, Taylor KI, et al; Working Group for the Evaluation of NHMRC Retinopathy Guideline Distribution. Changes in attitudes and practices of optometrists in their management of diabetic retinopathy after the release of NHMRC guidelines. Clin Experiment Ophthalmol 2001; 29: 121-124.

20 Wackerhausen S. Collaboration, professional identity and reflection across boundaries. J Interprof Care 2009; 23: 455-473.

21 Schuman JS. Glaucoma care: the patients perspective. What do patients want? Br J Ophthalmol 2008; 92: 1571-1572.

\section{Australia's most respected and comprehensive medical contact directory is online at www.mda.com.au}

Try it and see for yourself

For your obligation-free 30-day trial, contact (t) (02) 95626617

(e) mdasubs@ampco.com.au

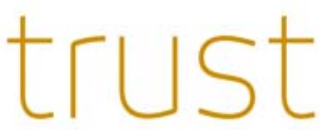

n 